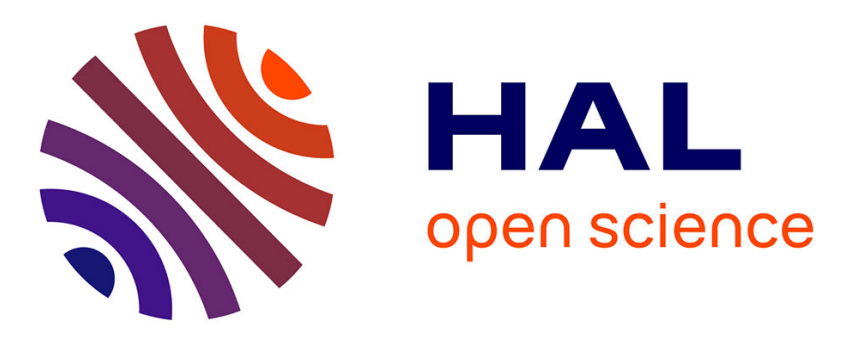

\title{
A Vector Approach to Transient Signal Processing
}

Florin-Marian Birleanu, Ion Candel, Cornel Ioana, Cedric Gervaise,

Alexandru Serbanescu, Gheorghe Serban

\section{To cite this version:}

Florin-Marian Birleanu, Ion Candel, Cornel Ioana, Cedric Gervaise, Alexandru Serbanescu, et al.. A Vector Approach to Transient Signal Processing. ISSPA 2012 - 11th International Conference on Information Science, Signal Processing and their Applications, Jul 2012, Montreal, Canada. pp.11741179. hal-00723308

\section{HAL Id: hal-00723308 https://hal.science/hal-00723308}

Submitted on 8 Aug 2012

HAL is a multi-disciplinary open access archive for the deposit and dissemination of scientific research documents, whether they are published or not. The documents may come from teaching and research institutions in France or abroad, or from public or private research centers.
L'archive ouverte pluridisciplinaire HAL, est destinée au dépôt et à la diffusion de documents scientifiques de niveau recherche, publiés ou non, émanant des établissements d'enseignement et de recherche français ou étrangers, des laboratoires publics ou privés. 


\title{
A VECTOR APPROACH TO TRANSIENT SIGNAL PROCESSING
}

\author{
Florin-Marian Birleanu ${ }^{\star}$, Ion Candel ${ }^{\star}$, Cornel Ioana ${ }^{\star}$,Cedric Gervaise ${ }^{\star}$, \\ Alexandru Serbanescu ${ }^{\ddagger}$, and Gheorghe Serban ${ }^{\circ}$ \\ ${ }^{\star}$ GIPSA-Lab / DIS, Grenoble Institute of Technology, France \\ E-mail: florin-marian.birleanu@gipsa-lab.grenoble-inp.fr \\ ‡ Military Technical Academy, Romania \\ ${ }^{\circ}$ University of Pitesti, Romania
}

\begin{abstract}
The detection and characterization of burst signals are challenging tasks for time-frequency analysis, due to their very short duration. This paper investigates in this context the recurrence plot analysis (RPA) method, from which it derives the vector samples processing (VeSP) concept. The paper shows that VeSP is a generic framework that unifies signal processing concepts like histogram and autocorrelation, which it also generalizes and extends. Results of VeSP based tools are provided, concerning detection of transient signals, noise reduction, and frequency estimation.
\end{abstract}

\section{INTRODUCTION}

Transient signals having a short time span are very common in signal processing applications. Telecommunications, reflectometry, ultrasonic testing, biomedical engineering, as well as the study of marine mammals are only some of the applications where transient signals play a key role. The common feature of all these transients is their short duration. Basically, any signal that we operate with in practice is a transient one (due to its finite duration). But we call transients those signals whose lengths are much shorter than the length of the entire signal being observed. This characteristic is the reason why transient signal detection and characterization are some challenging tasks. That is, it is challenging to efficiently detect the time intervals where transients are present, as well as to discriminate between various types of transients. Although time-frequency and time-scale tools offer interesting solutions to these signal processing problems, new approaches are being investigated in order to overcome the problems raised by the small number of samples in the transients. It was shown recently that the recurrence plot analysis (RPA) method [1] offers promising results in transient signal detection [2] and characterization [3]. The method [4, 5] is basically based on turning the (scalar) signal into a (multidimensional) trajectory, and then computing the recurrence plot [6] and quantifying the recurrences [7].

This paper approaches the RPA methodology from a signal processing point of view. The vector samples processig (VeSP) concept is introduced in Section 2 and in
Section 3 two signal processing tools (i.e. the time-distributed histogram (TDH), and the average magnitude difference function (AMDF)) are formulated in terms of vector samples. The use of VeSP based tools in transient signals processing is discussed in Section 4. It addresses the problems of detection, noise reduction, and fundamental frequency estimation. Section 5 provides results under noise condtions and section 6 concludes the paper.

\section{VECTOR SAMPLES PROCESSING (VESP)}

The recurrence plot analysis method is based on building a phase space trajectory by using succesive samples from the time series to be studied [4]. (The operation is called time-delay embedding and is based on Takens' Theorem $[8,9]$.) This phase space trajectory is composed of vectors like $\vec{v}_{n}=(s[n], s[n+d], s[n+2 d], \ldots, s[n+(m-1) d])$, where the elements $s$ are taken from the time series, and $m$ (phase space dimension) and $d$ (time delay) are some integer parameters. For a continuous-time signal, $s(t)$, things remain similar - see Figure 1. The phase space vectors are computed as [5]:

$$
\vec{v}_{t}=(s(t), s(t+\tau), \ldots, s(t+(m-1) \tau)),
$$

with $\tau=d \cdot T_{s}$, where $T_{s}$ is the sampling period. On each of the $m$ components of the vector $\vec{v}_{t}$ we have the signal to be analyzed, shifted in time with an integer multiple of the time delay parameter, $\tau$. We obtained this way the phase space representation of the signal. Practically, the vector $\vec{v}_{t}$ contains some of the values of signal $s$, taken equidistantly from the time interval $[t, t+w]$, where $w=(m-1) \cdot \tau$, as it can be seen in Figure 1. Therefore, the whole information contained in $\vec{v}_{t}$ can be found in the signal fragment located between the time instants $t$ and $t+w$.

Based on these considerations, we define the vector samples version of signal $s$ as:

$$
s_{i}^{(w)}(t)=s(i+t) \cdot W_{w}(i)
$$

where $w$ is the length of the vector samples, and $W_{w}$ is a rectangular window defined as:

$$
W_{w}(x)=\left\{\begin{array}{l}
1, \text { if } x \in[-w / 2, w / 2] \\
0, \text { otherwise }
\end{array} .\right.
$$




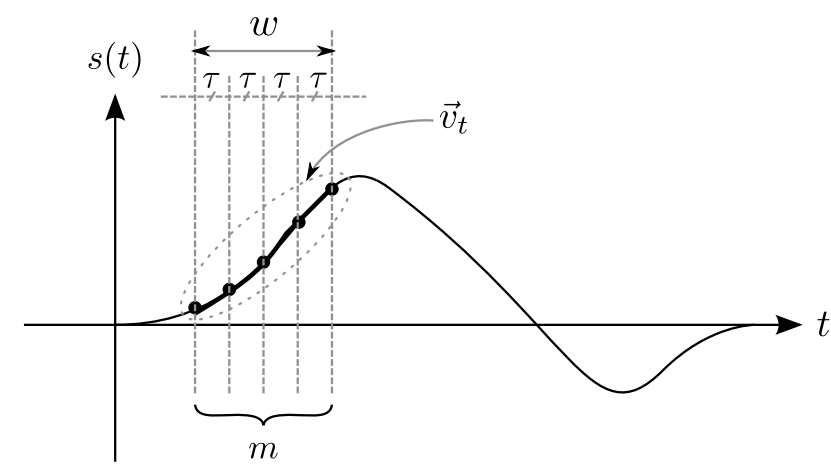

Fig. 1. Illustration of phase space vectors building in RPA. The $m$ components of a phase space vector span a time interval of length $w$.

We note that the $i$ index has the same significance and range of values as the $t$ argument, i.e. time. In fact, $s^{(w)}$ is a function of two arguments, $t$ and $i$, but we put $i$ as an index in order to simplify notations.

After computing the phase space representation of the time series, the next step in RPA consists in computing the recurrence plot $[6,4]$. This is done by comparing the distances between all possible pairs of phase space vectors. Recurrences are identified when such distances are below a certain threshold. In terms of vector samples, the mathematical expression for the recurrence plot is:

$$
R_{l, c}^{(w, \rho)}=\Theta\left(\rho-\mathcal{D}\left\{s_{i}^{(w)}(l), s_{i}^{(w)}(c)\right\}\right),
$$

where $\Theta$ is the Heaviside step function, $\rho$ is the recurrence threshold, and $\mathcal{D}$ denotes a method for computing the distance between two vector samples. For instance, when working with the Euclidean distance, $\mathcal{D}$ is defined as:

$$
\mathcal{D}\left\{s_{i}^{(w)}(l), s_{i}^{(w)}(c)\right\}=\left\|s_{i}^{(w)}(c)-s_{i}^{(w)}(l)\right\|
$$

with the norm of a vector sample $s_{i}^{(w)}(t)$ being defined as:

$$
\begin{gathered}
\left\|s_{i}^{(w)}(t)\right\|=\sqrt{<s_{i}^{(w)}(t), s_{i}^{(w)}(t)>} \\
=\sqrt{\int_{-\infty}^{+\infty} s_{i}^{(w)}(t) \cdot s_{i}^{(w)}(t) \mathrm{d} i .}
\end{gathered}
$$

However, $\mathcal{D}$ can be virtually any operator that produces a scalar value from two vector samples of the signal.

\section{VESP BASED SIGNAL PROCESSING TOOLS}

Starting from the recurrence plot defined in (4), several measures can be obtained. Two such measures are shown in Figure 2. The $\sigma_{c}$ measure is obtained by computing the normalized sum of the columns in $R$, while $\sigma_{d}$ is obtained by computing the normalized sum of the diagonals in $R$. They will be detailed in the following two subsections.

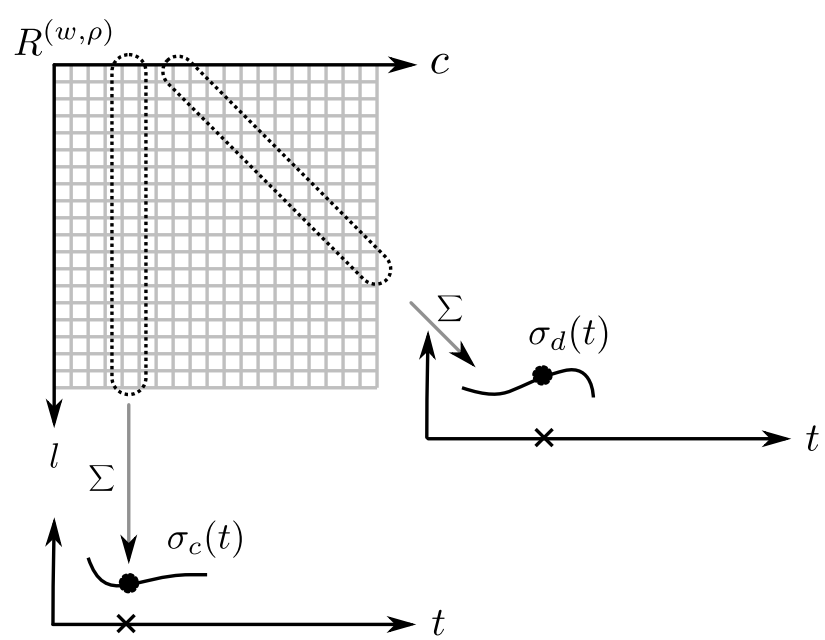

Fig. 2. Obtaining the $\sigma_{c}$ and $\sigma_{d}$ VeSP measures from the recurrence plot computed from signal vector samples of length $w$.

\subsection{Extended time-distributed histogram}

We define the $\sigma_{c}$ measure for the continuous-time signal $s$ as:

$$
\sigma_{c}^{(w, \rho)}(t)=\lim _{T_{\infty} \rightarrow \infty} \frac{1}{2 T_{\infty}} \int_{-\infty}^{+\infty} R_{l, t}^{(w, \rho)} \mathrm{d} l,
$$

where $R^{(w, \rho)}$ is the recurrence plot computed from signal $s$ using vector samples of length $w$ and a threshold of $\rho$. If $s$ is a discrete-time signal of $N$ samples, the previous equation becomes:

$$
\sigma_{c}^{(w, \rho)}[n]=\frac{1}{N} \sum_{l=1}^{N} R_{l, n}^{(w, \rho)} .
$$

This equation is sketched in a graphical form in Figure 2.

Having in mind that $\sigma_{c}[n]$ counts all recurrences around the vector sample located at time instant $n$, it can be noticed that when the vector sample $s_{i}^{(w)}[n]$ is reduced to the sample $s[n]$ (that is, when $w=0$ ), $\sigma_{c}^{(0, \rho)}[n]$ contains the (normalized) histogram of signal $s$, evaluated in $s[n]$. Hence, $\sigma_{c}$ is an extension of the time-distributed histogram of signal $s$ (as we have already shown in [2]).

\subsection{Extended average magnitude difference function}

We define the $\sigma_{d}$ measure for the continuous-time signal $s$ as:

$$
\sigma_{d}^{(w, \rho)}(t)=\lim _{T_{\infty} \rightarrow \infty} \frac{1}{2 T_{\infty}} \int_{-\infty}^{+\infty} R_{l, l+t}^{(w, \rho)} \mathrm{d} l .
$$

If $s$ is a dicrete-time signal of $N$ samples, this equation becomes:

$$
\sigma_{d}^{(w, \rho)}[n]=\frac{1}{N-t} \sum_{l=1}^{N-t} R_{l, l+n}^{(w, \rho)} .
$$

Figure 2 sketches this equation in a graphical form. 


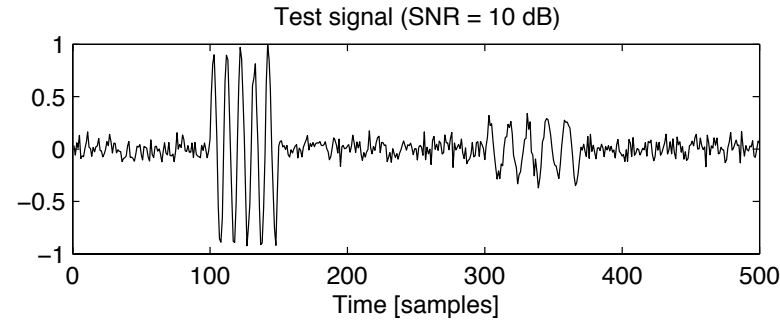

Fig. 3. The test signal: two sinusoidal bursts with different amplitudes and frequencies.

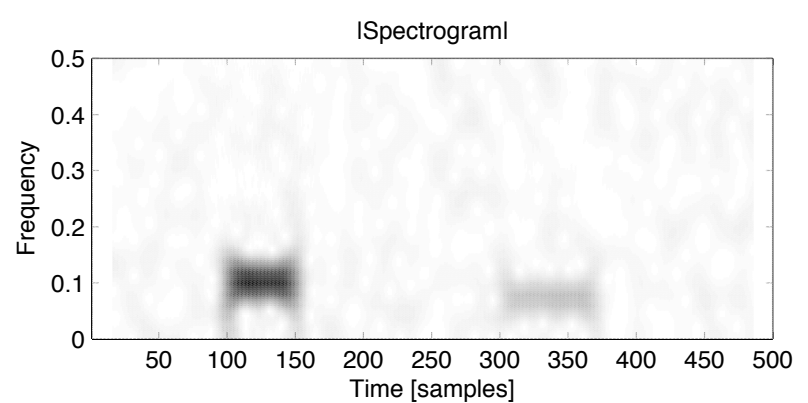

(a)

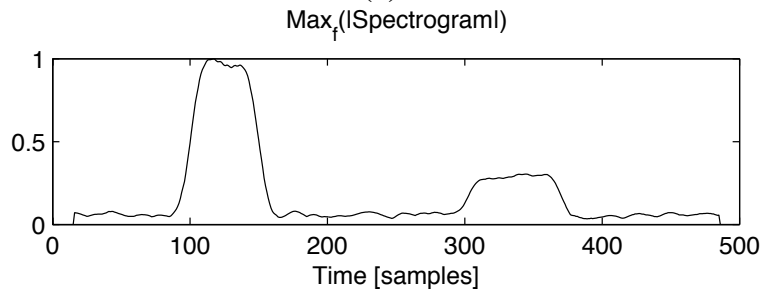

(b)

Fig. 4. (a) Spectrogram of the test signal. (b) Detection curve obtained by taking the maximum frequency in the spectrogram for each time instant.

When the vector sample $s_{i}^{(w)}[n]$ in reduced to the sample $s[n]$, the previous equation becomes:

$$
\sigma_{d}^{(0, \rho)}[n]=\frac{1}{N-t} \sum_{l=1}^{N-t} \Theta(\rho-|s[l+n]-s[n]|) .
$$

The similarity of this equation to the expression of the average magnitude difference function (AMDF) [10] is obvious. We recall that the AMDF for signal $s$ is computed as follows:

$$
A M D F[n]=\frac{1}{N-n} \sum_{l=1}^{N-n}|s[l+n]-s[n]| .
$$

Equation (11) extends $A M D F$ in (12) by binarizing the differences using the threshold $\rho$, and Equation (10) further extends it by replacing samples with vector samples of length $w$.

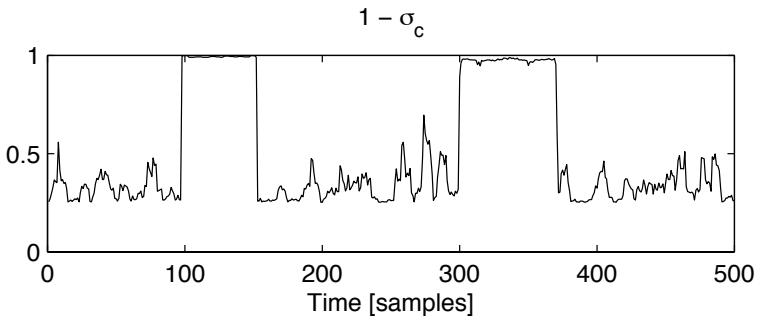

Fig. 5. Detection curve obtained for the test signal by computing the VeSP measure $\overline{\sigma_{c}}\left(7, \rho_{0}\right)$.

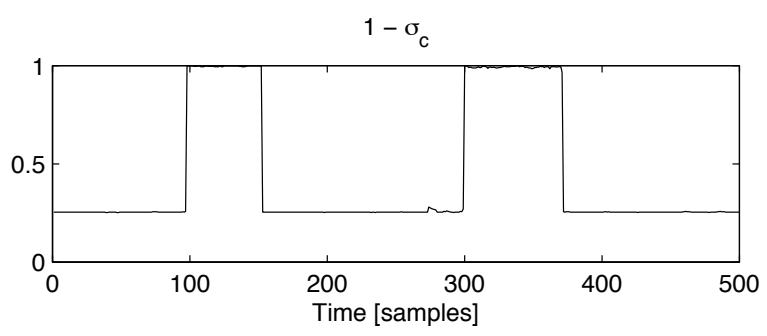

(a)

Signal * $\left(1-\sigma_{c}\right)$

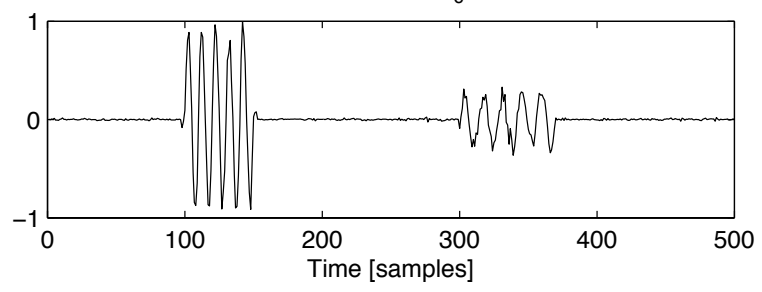

(b)

Fig. 6. (a) Detection curve obtained by using $\bar{\sigma}_{c}$, after two iterations. (b) Denoised signal obtained by multiplicating the analyzed signal with the obtained detection curve, after the second iteration.

\section{TRANSIENT SIGNAL PROCESSING USING VESP BASED TOOLS}

Let us consider the test signal in Figure 3. It contains two transients having different start times, durations, and frequencies. This kind of signals may appear in various applications (e.g. ultrasonic testing, telecommunications) where the common point consists in emitting short burst signals that propagate through a certain environment and finally reach the receiver. Reflexions and multipath propagation may lead to different delays and attenuations in the received signal. Regardless of which is the exact application, for a signal like the one in Figure 3 we are interested in estimating the different parameters of the transients it contains.

Figure 4 shows the solution offered by the spectrogram to the problem of transients detection. It should be noted that the quality of the results depends strongly on the size of the analysis window. For obtaining the results shown in the figure, we used a window size of 31 samples with an overlapping of 30 samples. It can be noticed that the obtained detection curve indicates quite well the positions of the two transients. However, it is difficult to properly 


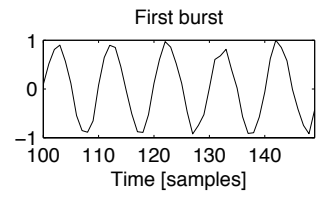

$\sigma_{d}$
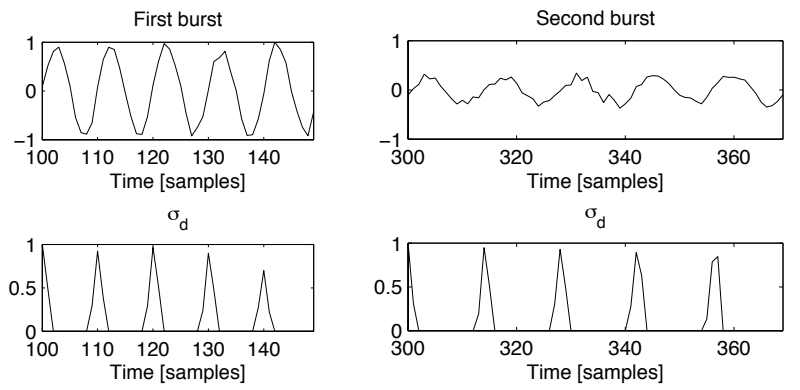

$\sigma_{\mathrm{d}}$

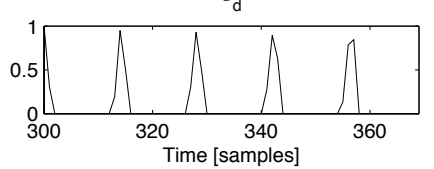

Fig. 7. The VeSP measure $\sigma_{d}$, computed for the two transients in the test signal.

choose a detection threshold, due to the low values obtained for the second transient. The VeSP measure $\sigma_{c}$ that we introduced in the previous section offers a potential solution to this problem, as Figure 5 shows. The detection curve is obtained by taking the complement of $\sigma_{c}$, that is:

$$
\bar{\sigma}_{c}^{(w, \rho)}[n]=1-\sigma_{c}^{(w, \rho)}[n] .
$$

It detects the second transient very well, regardless of its low amplitude. We used a value of 7 for the vector sampling window $(w)$, and for the $\rho$ threshold we used the average distance between all the successive vector samples of the signal, that is:

$$
\rho_{0}=\frac{1}{N-1} \sum_{l=1}^{N-1}\left\|s_{i}^{(w)}[l+1]-s_{i}^{(w)}[l]\right\| .
$$

However, the problem of choosing a detection threshold is still not completely solved by $\bar{\sigma}_{c}$. Choosing a threshold of 0.5 would not miss any of the two transients (unlike in Figure 4.(b)), but would also generate some false positives. The fact that the two transients are properly detected (i.e. values close to 1 in the detection curve) suggests that the detection curve in Figure 5 could be improved by multiplicating it with the original signal (thus reducing the noise) and reiterating the computation of $\bar{\sigma}_{c}$. Figure 6.(a) shows the detection curve obtained after only two such iterations. It is much cleaner than the one in Figure 5. This holds true also for the denoised signal (Figure 6.(b)).

In addition to detection, we might also be interested in estimating the frequencies of the two transients. The VeSP measure $\sigma_{d}$ can be useful for this purpose, as Figure 7 shows. It can be noticed that $\sigma_{d}$ has sharp equally spaced maxima, and the distance between these maxima is the fundamental period of the analyzed burst signal. Hence, the estimation of this distance leads to an estimation of the fundamental frequency.

\section{RESULTS}

Figure 8 shows in a compact form the behaviour under noise conditions of the detection curves illustrated in Figures 4.(a) and 5. Each line in the three plots corresponds to a certain signal-to-noise ratio, as indicated on the vertical axis. The minimum values are represented in white,

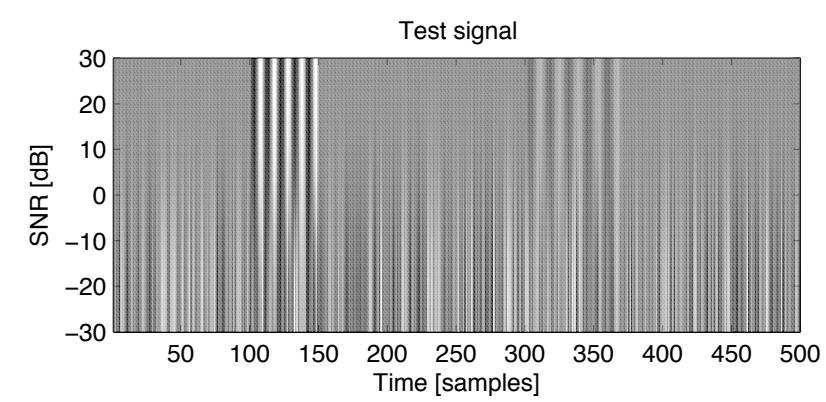

(a)

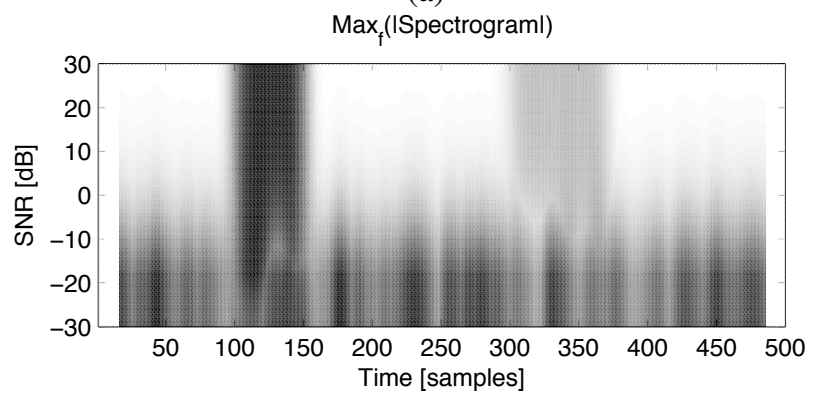

(b)

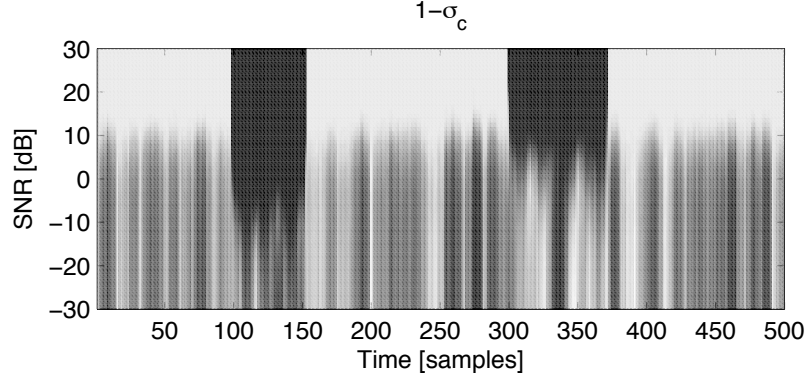

(c)

Fig. 8. (a) The test signal in Figure 3, for multiple signalto-noise ratios. (b) Detection curves obtained with the spectrogram. (c) Detection curves obtained with $\overline{\sigma_{c}}$.

while the maximum values are represented in black. Additive white Gaussian noise was used, and the signal-to-noise ratio was computed as:

$$
S N R_{[d B]}=20 \cdot \log _{10} \frac{\operatorname{rms}(S)}{\operatorname{rms}(N)},
$$

where $S$ is the signal, $N$ is the noise, and $\operatorname{rms}(\cdot)$ denotes the root mean square.

When detection and denoising are applied iteratively as discussed in the previous section, the results obtained after the fourth iteration are those shown in Figure 9.

Finally, Figure 10 shows a comparative view on the results given by AMDF [10] and $\sigma_{d}$ for the second transient in the test signal in Figure 3. We note that AMDF is represented using white for large values and black for small values, in order to facilitate the visual comparison with $\sigma_{d}$.

\section{CONCLUSION}

The results in the previous section show that vector samples based detection of transient signals performs better 


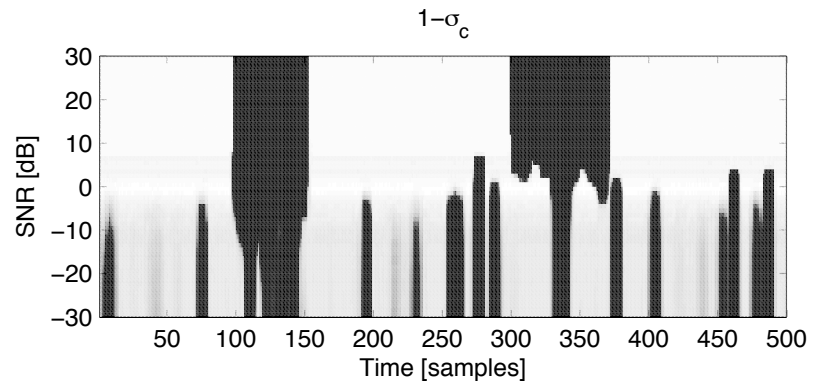

(a)

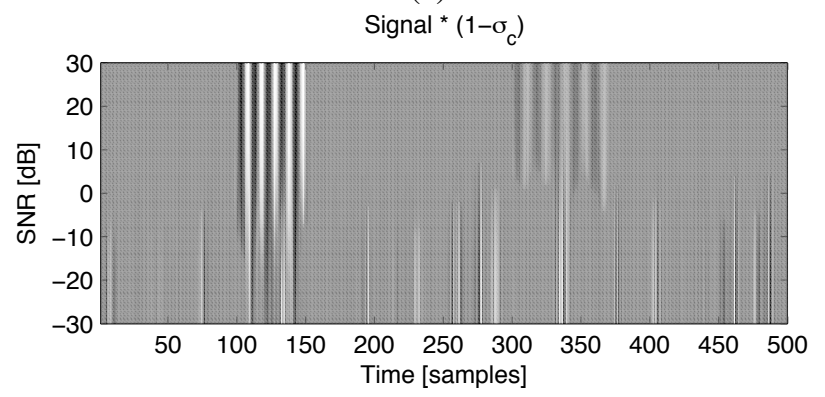

(b)

Fig. 9. (a) Detection curves obtained with $\bar{\sigma}_{c}$ from the test signals in Figure 8.(a), after four iterations. (b) Denoised signals, obtained after four iterations.

than spectrogram based detection. Due to its characteristics, the $\bar{\sigma}_{c}$ measure can also be used to reduce noise in the analyzed signal (by using point to point multiplications). We also showed that improvements under noise conditions can be obtained by recursively computing $\bar{\sigma}_{c}$ from the denoised signal. We showed as well that the $\sigma_{d}$ measure offers cleaner results than the average magnitude difference function.

Hence, the two vector samples processing measures presented in this paper proved to be useful in tasks such as transient signal detection, noise reduction, and fundamental frequency estimation. Further work could target a method for choosing the optimum value for the vector sampling window, as well as a deeper study on the influence of the recurrence threshold. Another research direction could explore various methods for defining the distance between two vector samples. Besides that, new measures based on vector samples computations could be explored as well.

\section{REFERENCES}

[1] N. Marwan, "A historical review of recurrence plots," The European Physical Journal Special Topics, vol. 164, pp. 3-12, 2008.

[2] F.-M. Birleanu, C. Ioana, A. Serbanescu, and J. Chanussot, "A time-distributed phase space histogram for detecting transient signals," in IEEE International Conference on Acoustics, Speech and Signal Processing, Prague, Czech Republic, 22-27 May 2011, pp. 3844-3847.

[3] F.-M. Birleanu, C. Ioana, C. Gervaise, A. Serbanescu,

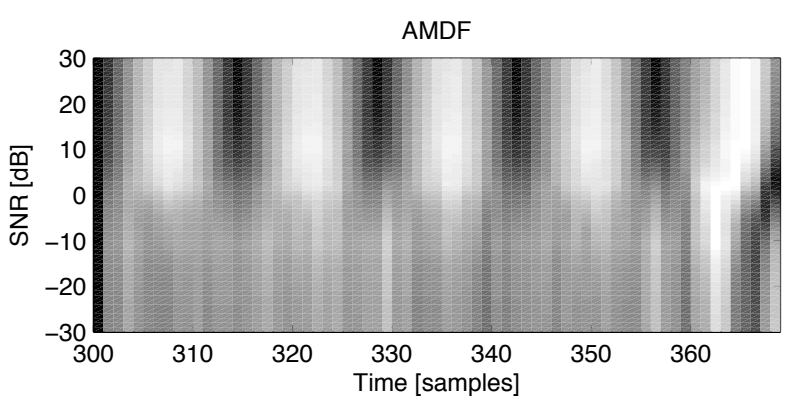

(a)

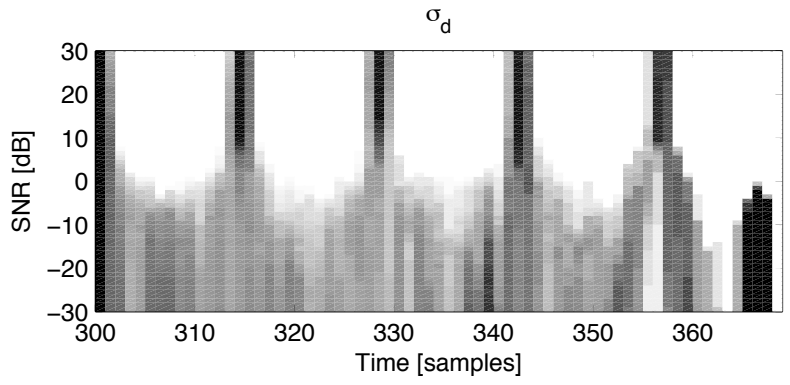

(b)

Fig. 10. (a) The average magnitude difference function, computed for the second transient in Figure 3 at multiple signal-to-noise ratios. (b) The $\sigma_{d}$ VeSP measure, computed for the same signals.

and J. Chanussot, "Caractérisation des signaux transitoires par l'analyse des récurrences de phase," in Gretsi'11 - 23ème colloque sur le traitement du signal et des images, Bordeaux, France, 5-8 September 2011.

[4] N. Marwan, M. Carmen Romano, M. Thiel, and J. Kurths, "Recurrence plots for the analysis of complex systems," Physics Reports, vol. 438, pp. 237329, January 2007.

[5] F.-M. Birleanu, C. Ioana, C. Gervaise, J. Chanussot, A. Serbanescu, and G. Serban, "On the recurrence plot analysis method behaviour under scaling transform," in IEEE Statistical Signal Processing Workshop, Nice, France, 28-30 June 2011, pp. 789-792.

[6] J.-P. Eckmann, S. Oliffson Kamphorst, and D. Ruelle, "Recurrence plots of dynamical systems," Europhysics Letters, vol. 4, no. 9, pp. 973-977, November 1987.

[7] J.P. Zbilut, A. Giuliani, and C.L. Webber Jr., "Recurrence quantification analysis as an empirical test to distinguish relatively short deterministic versus random number series," Physics Letters A, vol. 267, pp. 174-178, 2000.

[8] N. Packard, J. Crutchfield, D. Farmer, and R. Shaw, "Geometry from a time series," Physical Review Letters, vol. 45, no. 9, pp. 712-716, 1980.

[9] F. Takens, "Detecting strange attractors in turbulence," in Dynamical Systems and Turbulence. Lec- 
ture Notes in Mathematics, D. A. Rand and L.-S. Young, Eds., vol. 898, pp. 230-242. Springer-Verlag, 1981.

[10] G. Muhammad, "Extended average magnitude difference function based pitch detection," The International Arab Journal of Information Technology, vol. 8, no. 2, pp. 197-203, April 2011. 